\title{
WheelWalker: a foot-wheel driving exoskeleton for the alternate walk of paraplegic patients
}

\author{
Qingchuan Ma , Linhong Ji and Rencheng Wang \\ State Key Laboratory of Tribology, Department of Mechanical Engineering, Tsinghua University, Beijing 100084, China
}

\begin{abstract}
Upright walk is the fundamental need of paraplegic patients. In this study, we proposed a novel lower-limb exoskeleton for assisting the patients to walk alternately and further improve their engagement in the rehabilitation training. The exoskeleton was driven by a hub motor which was mounted under the foot, and a pair of custom-made crutch with wireless controller was used to control the walking condition of exoskeleton and maintain balance. This paper mainly introduced the general designing concept and detailed specifications of each modules of the exoskeleton. A pilot using experiment was performed on two healthy subjects to qualitatively analyse the capability of this exoskeleton when adopting different gaits. The results showed that the subjects with different body characteristics could complete continued walk by wearing this exoskeleton either in a three-point gait mode or four-point gait mode.
\end{abstract}

\section{Introduction}

Paraplegia affects the lives of millions of people and most of them have to rely on the conventional assistive device like wheelchair or reciprocating gait orthosis (RGO) to achieve the basic lives[1, 2]. However, longterm use of the wheelchair could induce several physical and psychological problems due to maintaining of the sitting posture[3]. And the physical energy consumption of RGO is too remarkable often leading to a relative high abandon rate[4]. Portable exoskeletons have become the promising intelligent rehabilitation devices to assist patients walking upright without causing problems that wheelchair and RGO have. Several successful prototypes were developed including ReWalk (Israel)[5], Ekso (USA)[6], WPAL (Japan)[7]. Whereas, the complicated controlling system, short battery time and the price still restrict current portable exoskeleton from the widely application in clinic[8-10].

From the perspective of mechanical structure, most of the current portable exoskeletons belong to joint-driving, some researchers believe that such mechatronic design have fundamental shortcoming and need to be revolutionary updated to satisfy the future requirement [11, 12]. Li et al also suggested that researchers in this area should downgrade the automation level and design the exoskeleton as a supplementary equipment rather than a robot-like machine[13]. These researches indicate that the development of a simple, reliable and affordable portable exoskeleton is a more practical way for the patientoriented clinical device.

Wheeled mechanism has better power efficiency and easier control experience than joint driving as it utilized in wheeled robot[14]. Therefore, based on the analysis of current rehabilitation study, we developed a foot-wheel driving exoskeleton for paraplegic patents which uses simplified mechatronic system to realize the basic needs of lower-limb disabled individuals for upright walking.

\section{System overall designing concept}

The main goal of this exoskeleton is using the simplest mechatronic part to realize the basic upright walking, furthermore, downgrading the automation level of exoskeleton and using the least sensor technologies to empower the user fully control of their walking procedure and improve their rehabilitation engagement. This system mainly consists of two parts as shown in Fig. 1, the footwheel driving exoskeleton and the wireless controlling crutch. The powered exoskeleton could immobilize and support user's body and drive the lower limb moving forward by the wheeled foot. The custom-made crutch could maintain the balance when walking and control the moving status of the exoskeleton by a wireless controller which was embed in the handlebar of the crutch.

The limitations for target users including: The user should have no spastic cerebral palsy to avoid the unpredictable falling; the user should have an abled upper limb to use the crutch and the paraplegia level of subjects should better than T10; the user's height and weight are less than $185 \mathrm{~cm}$ and $100 \mathrm{~kg}$, respectively.

Several basic functions should be satisfied according to the project planning:

(a) The exoskeleton should assist user to complete the independent walk in a standing posture on a level or inclined road at a maximum speed of $2.1 \mathrm{~km} / \mathrm{h}$; 
(b) The user could fully control the walking status in real time including speed, step length, stop and start;

(c) The exoskeleton's wheel should automatically maintain a secure stop when there is no signal received from the crutch and the maximum step length also should be limited to protect the patient from the potential electronic system failures or misoperations;

(d) The length of the mechanical frame should be adjustable according to the specific body characteristic of the user.

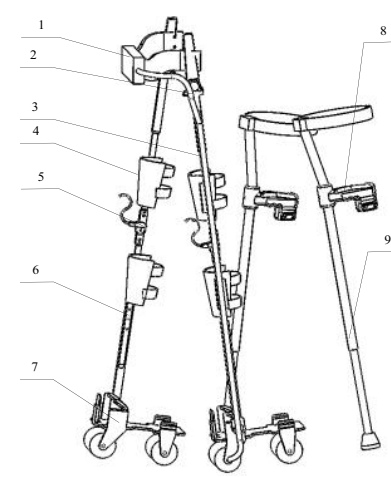

(a)

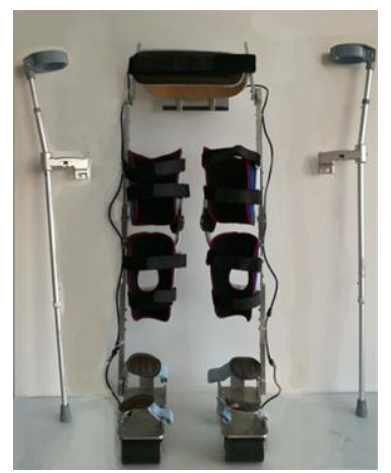

(b)
Figure 1. The 3D designing model (a) and front appearance of the exoskeleton system (b).

\section{Technological details}

\subsection{Mechanical specifications}

The hip and ankle of this exoskeleton have one rotatory degree of freedom in sagittal plane respectively, while the knee joint can be manually locked or unlocked in standing or sitting posture. The degree of freedom is a crucial issue in the design of rehabilitation device as it can directly affect the performance of an exoskeleton. Here we developed this full Hip-Knee-Ankle-Foot (HKAF) exoskeleton which only enable the rotation of joint in sagittal plane while mechanically restrict movement in coronal plane.

Typically, human has a $15^{\circ}$ of maximum ankle dorsiflexion angle and $10-20^{\circ}$ of plantar flexion angle at the normal walking gait. Therefore we limited the maximum forward and backward rotation of this exoskeleton's ankle at $15^{\circ}$ separately for safety reason. For a better adaption in users with different height, the length of the exoskeleton's shank could be adjustable in a range of $70 \mathrm{~mm}$.

Different materials were used at different part of this exoskeleton to balance the weight and the strength. The exoskeleton's thigh and shank part were made of highstrength steel bars to withstand the supporting load of user. The pelvis, hip and ankle were all made by aluminium alloy. While the knee joint was made by steel as it has to provide a secure lock of the lower-limb. The knee fixator was made of PE (polyethylene) to achieve a light and high-stiffness supporting of leg, while sponge cloth was installed at the inner surface of this fixator enabling adequate comfortable using experience.

\subsection{Foot-wheel driving unit}

The frame of the exoskeleton's foot consisted of six aluminium plates and had two Velcro bands to securely fix the foot of user (Fig.2). The ankle joint of the exoskeleton connected the foot with two thrust bearings and a custom-made connecting bolt. We made a restricting groove at ankle bar and used a restricting bolt at the rear plate of the foot to limit the total rotation angle of the ankle in a range of $30^{\circ}$. Eight screwed holes with a $10 \mathrm{~mm}$ interval were designed at the ankle joint bar to provide $70 \mathrm{~mm}$ adjustable length for the different height of users.

Each foot-wheel driving unit uses two wheels, the front one is unpowered assistant wheel and the rear one is a custom-made powered hub motor. The hub motor is specially designed and manufactured for this study, which adopted an external BLDC motor and two-level planetary reducer to achieve a high-torque and smalldiameter performance. As shown in Fig. 2(b), the hub motor was covered by $8 \mathrm{~mm}$ thick anti-slip rubber with a final rotation speed of $150 \mathrm{r} / \mathrm{min}$, which is $2.1 \mathrm{~km} / \mathrm{h}$ in linear moving, approaching the maximum speed of ReWalk $(2.6 \mathrm{~km} / \mathrm{h})$. The high reducing rate of this hub motor could enable a remarkable stopping torque functioning as the brake. Cooperated with the custommade motor controller, in our test this hub motor could realize a reliable stop.

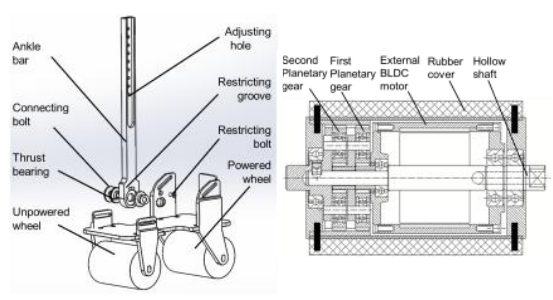

(a) (b)

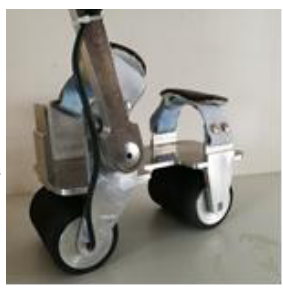

(c)
Figure 2. The detailed 3D designing model (a), section view of the hub motor (b), and the overall appearance (c) of the footwheel driving unit. In 3D model, the fixing bands were removed for a better illustration of the mechanical details.

\subsection{Wireless controlling crutch}

A pair of custom-made arm crutch were used in this system to provide balance maintaining and control of exoskeleton. The handlebar of this crutch was made by aluminium alloy which mainly consisted of three parts, the upper shell, the lower shell and the button (Fig. 3). The upper shell could provide the weight support for the balance maintaining and a wireless controller was embedded in it. The lower shell covered the wireless controller and had a higher platform at the end of handlebar to protect the button from the misoperation of the user. The button had a slope with $30^{\circ}$ of inclination which could transfer the rotatory speed control of joystick into a $20 \mathrm{~mm}$ linear control. The button could slide in the guiding slot of lower shell and would be recoiled by a 
spring. The handlebar was mounted at the upper body of crutch and the length of this crutch could be adjusted according to the height of the user.

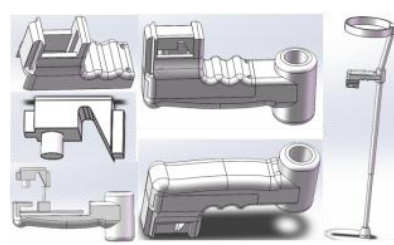

(a)

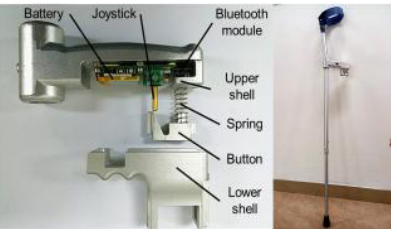

(b)
Figure 3. The detailed 3D designing model (a) and explosive view (b) of the wireless controlling crutch.

\subsection{Electronic system}

Fig. 4 showed the electronic modules we utilized in this prototype. The wireless controller is a STM32F103 based Bluetooth transmitter which is powered by a rechargeable $4.2 \mathrm{~V}^{*} 630 \mathrm{~mA}$ Li-ion battery. The joystick is the crucial part of the controller which could control the moving status of the exoskeleton. The rotational angles of joystick directly reflect to the speed of motor and the brake will be enabled when the joystick maintain the 0 degree. The wireless receiver has a same Bluetooth module with the transmitter, which could output the PWM signal to the motor controller for speed control. The motor controller is a custom-made driver for a $24 \mathrm{~V}$ Hull-sensor based BLDC motor. When the PWM signal was detected, this controller will actuate the motor, otherwise it will induce an electronic brake. A $24 \mathrm{~V}^{*} 5000 \mathrm{mAH}$ rechargeable Li-ion battery pack was chosen as energy source to power the exoskeleton. All electronic parts were packaged in a $220 * 122 * 32 \mathrm{~mm}$ aluminium box which was mounted at the pelvis of the exoskeleton. The wire of motor was fixed along the mechanical frame and connected the motor controller at the lateral side of electronic box with the aviation plugs.

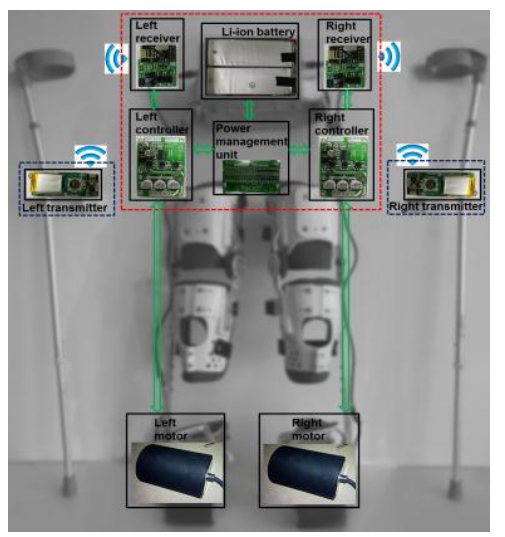

Figure 4. Electronic system block diagram (the circuit parts were enlarged). The red dashed box indicates the electronic parts in the main electronic box, blue dashed box indicates the electronic parts in the crutch, green linking arrow indicates the wire connection among parts.

\section{Pilot tests on healthy subjects}

To qualitatively analyse the capability of this exoskeleton, we conducted the indoor using tests on healthy subjects. The preparing procedure should be finished before functionally operating this exoskeleton. First, the user should wear the equipment at sitting posture, unlocking the knee joint of the exoskeleton and immobilizing the lower limbs with the fasten band. Secondly, switch on the exoskeleton and crutch, then stand up and lock the knee joint. Thirdly, press the button of crutch by the index finger to control the leg moving forward.

The main testing objective for this exoskeleton is its usability when adopting the four-point gait and threepoint gait, which frequently appeared in the common crutch users. As shown in the Fig. 5, the motion sequence of the four-point gait is that the left crutch move forward first and the right leg step later, then the right crutch move forward and the left leg step, then repeat this sequence. In three-point gait, the two crutches move forward simultaneously and step left leg later, then crutches move forward again and step the right leg, then repeat this sequence. Although the foot will keep touch the ground in the whole moving procedure in this exoskeleton, while like the normal crutch user the leg of the exoskeleton would also step alternatively to walking forward. Therefore, theoretically either four-point gait or three-point gait could be adopt in this exoskeleton.
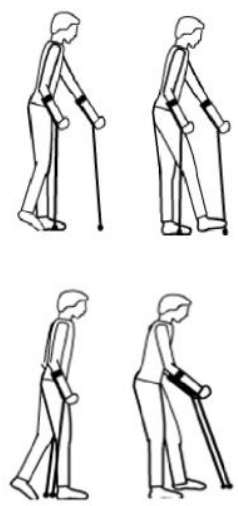
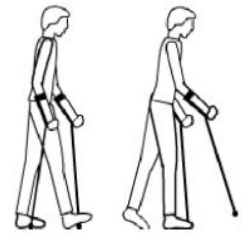

(a)

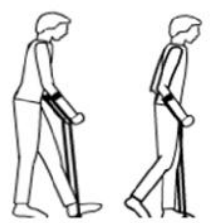

(b)
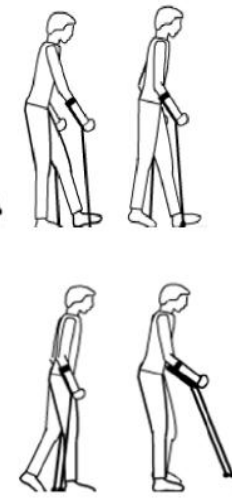

Figure 5. The typical operational procedure of the crutch when adopting four-point gait (a) and three-point gait (b)[15].

As shown in Fig. 6a, a healthy male subject with the height and weight $180 \mathrm{~cm}, 70 \mathrm{~kg}$ was recruited to operate the exoskeleton in four-point gait mode. The user moved the right crutch first and then pressed the button to control the left leg moving forward, and then repeated this procedure in right leg; A healthy subject with the height and weight $168 \mathrm{~cm}, 60 \mathrm{~kg}$ tested the three-point gait mode (Fig. 6b). Similarly with the common crutch user, the subject moved two crutches forward and then pressed one of the controlling button to move one leg, then repeated this procedure to move another leg.

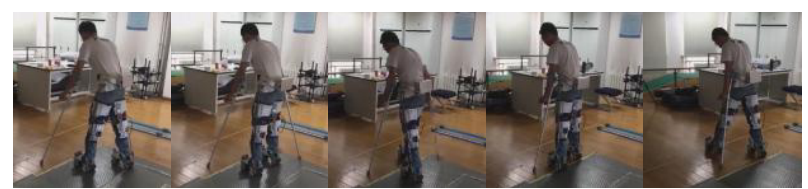

(a) 


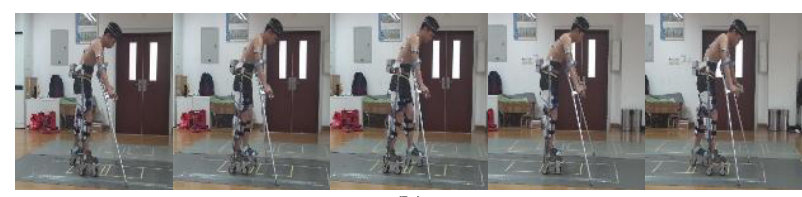

(b)

Figure 6. Snapshots of using experiments on two healthy subjects with four-point gait (a) and three-point gait (b).

It should be noted that we tested two different gait modes by different subjects mainly for the consideration of testing diversity, while the effectiveness of different operation modes will not be affected by the different physical characteristics of user. Our test showed that both the user could individually operating the exoskeleton in four-point gait mode or three-point gait mode without the help from others. An additional fact we found in our tests is that although the capability of length adjusting for this exoskeleton's shank is $70 \mathrm{~cm}$, whereas the wider scope of application than the range of $70 \mathrm{~mm}$ is still acceptable. This most because the exoskeleton do not need to consider about the lower limb alignment as joint-driving exoskeleton since the knee joint is locked when walking. In our practicing test, both the subjects with the height of $180 \mathrm{~cm}$ and $168 \mathrm{~cm}$ could operate this exoskeleton freely without uncomfortable using experience.

\section{Conclusions}

Upright walk is the most desirable needs of paraplegic patients. In this study we proposed a wheeled foot exoskeleton which uses a custom-made hub motor as driving unit and the crutch based wireless controller for controlling, enabling the patient to walk in a standing posture. The general designing concept and detailed specifications of each module were presented in this paper. Pilot experiments on two healthy subjects were performed and the results showed that the user could successfully complete the individual upright walking. In future study, we plan to perform a motion analysis experiment in a 3D gait analysis system to obtain more objective and evaluable data of this exoskeleton.

\section{References}

1. S. Hussain, Q. X. Sheng, P. K. Jamwal, J. Parsons, Med. Eng. Phys., 34, 1448-1453 (2012)

2. R. E. Cowan, B. J. Fregly, M. L. Boninger, L. Chan, M. M. Rodgers, D. J. Reinkensmeyer, J. Neuroeng. Rehabil, 9, 971-981 (2012)

3. A. Kittel, M. A. Di, H. Stewart, Disabil. Rehabil. 24, 106-114 (2009)

4. V. Castellano, D. Coratella, and F. Felici, J.Electromyogr. Kines. 9, 149-157(1999)

5. S. Hussain, S. Q. Xie, P. K. Jamwal, J. Parsons, Med. Eng. Phys., 34. 1448-1453 (2012)

6. K. A. Strausser, T. A. Swift, A. B. Zoss, H. Kazerooni, B. C. Bennett, K. A. Strausser, ASME 2011 Dynamic Systems and Control Conference and Bath/ASME Symposium on Fluid Power and Motion Control, 419-425 (2011)

7. S. Tanabe, S. Hirano, E. Saitoh, NeuroRehabilitation, 33, 99-106(2013)

8. K. H. Low, Defense Science Research Conference and Expo, 1 - 10 (2011)

9. R. Bogue, Industrial Robot, 42, 5-10(2015)

10. JL. Contreras-Vidal, A. B. N, J. Brantley, JG CruzGarza, Y. He, J. Neural Eng., 13, 3(2016).

11. D. Sasaki, T. Noritsugu, M. Takaiwa, IEEE/RSJ International Conference on Intelligent Robots and system, 4440-4445 (2013)

12. J. L. Contreras-Vidal and R. G. Grossman, IEEE, 579-1582013(2013)

13. Z. Li, H. Xie, W. Li, Z. Yao, Chin. J. Mech. Eng-En, 27, 437-447(2014)

14. R. P. M. Chan, K. A. Stol, C. R. Annu. Rev. Control, 37, 89-103(2013)

15. Z. Yang, Gait planning and human-machine coordination study of walk assisting exoskeleton for paraplegics (Master Thesis, Mechanical Engineering, Tsinghua University, Beijing, 2014). 